\section{Proton Tunnelling in Intermolecular Hydrogen Bonds}

Tony Horsewill

University of Nottingham, United Kingdom

Mark Johnson

Institut Laue-Langevin, Grenoble, France

Hans Peter Trommsdorff

Director of Research, Université Joseph Fourier, Grenoble, France

The wavefunctions of particles extend beyond the classically accessible regions of potential energy surfaces (PES). A manifestation of this partial delocalization is the quantum mechanical tunnelling effect which enables a particle to escape from a metastable potential well. Barrier controlled atomic rearrangements and chemical reactions can thus occur at $0^{\circ} \mathrm{K}$ in the absence of thermal activation. Tunnelling is most important for the lightest atoms, so that the determination of its contribution to proton transfer, one of the most fundamental chemical reactions, is an important issue. We have employed quasi-elastic neutron scattering (QENS) and nuclear magnetic resonance (NMR) techniques as well as optical spectroscopy to study the motion of protons in the hydrogen bond of benzoic acid crystals, a system which has emerged as a particularly suitable model since proton transfer occurs in a near symmetric double well potential. The influence of quantum tunnelling was revealed and investigated in these experiments. This work provides a benchmark for theoretical descriptions of translational proton tunnelling.

Proton transfer reactions along hydrogen bonds are ubiquitous and of fundamental importance to many chemical and most biological systems, where proton transfer inter-converts different possible tautomer structures of a molecule (eg nucleic acidbase pairs). The observation of large H/D isotope effects and of temperature independent reactions rates at very low temperatures indicate that tunnelling contributes to these reactions. A proper quantitative description of these, of the

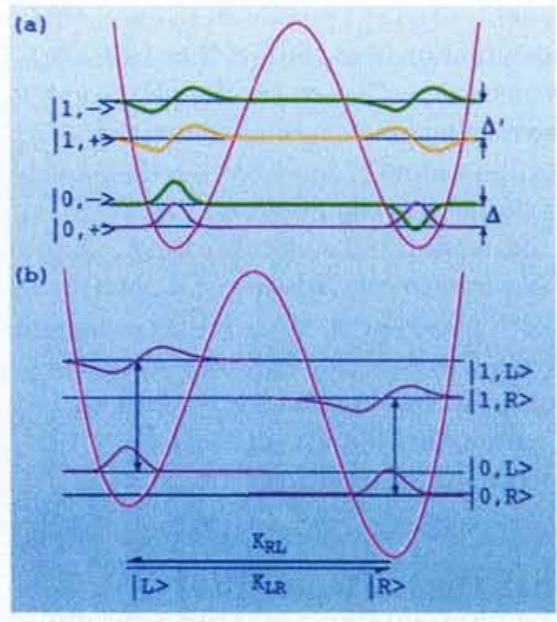

Fig 1 Manifestation of coherent and incoherent tunnelling in symmetric and asymmetric doubleminimum potentials (DMP). Only the four lowest energy-states are indicated.

a) Coherent tunnelling in a symmetric DMP is manifested by the $|+>|-,>$ (tunnelling) splitting of the vibrational states, $|0>| 1>,, \ldots$

b) In asymmetric DMP, vibrational states are localized in either well. Thermal equilibrium within each well is established by fast intrawell relaxation $|0\rangle \leftrightarrow|1\rangle$, while incoherent tunnelling is manifested by the slow interwell $|\mathrm{L}>\leftrightarrow| \mathrm{R}>$ relaxation

relevant PES and, at higher temperatures, the issue of the contribution of tunnelling to the reaction rate remain subjects of intense current research. The principal difficulty lies in the fact that the proton displacement is accompanied by an electronic rearrangement, which results in minor readjustments of the equilibrium positions of the other atoms of the molecule and the environment. Because of this coupling, proton transfer must be described within a multidimensional PES.
While the force field of the molecule, as derived from vibrational spectroscopic data (infrared, Raman, inelastic neutron scattering) gauges the PES near the equilibrium positions, tunnelling provides information about the barrier region separating potential minima. Tunnelling is quantitatively best characterized by the observation of a splitting of the otherwise degenerate levels localized in either well of a symmetric double minimum potential ('coherent' tunnelling, fig 1a). This tunnelling splitting, $\Delta$, depends exponentially on the mass, $\mathrm{m}_{\mathrm{o}}$, of the tunnelling particle and the properties (width d, height $\mathrm{V}_{\mathrm{o}}$ ) of the barrier separating potential minima as follows: $\log (\Delta) \approx-\mathrm{d}\left(\mathrm{m}_{\mathrm{o}} \mathrm{V}_{\mathrm{o}}\right)^{1 / 2}$. However, the coupling to the environment makes potential wells inequivalent ( fig $_{1} \mathrm{~b}$ ), with the consequence that coherent tunnelling is suppressed and, in the limit of very low temperatures, the protons become localized in the more stable well. Interwell relaxation can still be observed with a rate, which is determined by 'incoherent' tunnelling and which scales with $\Delta^{2}$.

Benzoic acid (fig 2) is the only compound in which both coherent and incoherent tunnelling of protons along a hydrogen bond has been measured in the limit of low temperatures by optical spectroscopic techniques [1-4]. These measurements are illustrated in fig 3 . A dye molecule, imbedded in the benzoic acid crystal perturbs slightly the PES governing the proton dynamics of neighbouring acid dimers. In the ground state of the dye, the energy difference, A, between the two tautomers, labelled L and $R$, is very small $(A<\Delta)$, the level splitting equals $\left(\mathrm{A}^{2}+\Delta^{2}\right)^{1 / 2}$, and the protons are delocalized over both wells. In the excited state of the dye, the potential becomes asymmetric and the protons are localized. The four optical transitions, which can be observed, are labelled a to $\mathbf{d}$ and are shown as vertical lines. The small splitting of the ground state levels is observed in the absorption spectra and is resolved by hole burning techniques. The values of $A$ and $\Delta$ can be determined from this splitting and the intensity ratio of the two neighbouring transitions as follows

$\mathrm{I}(\mathbf{a}) / \mathrm{I}(\mathbf{b})=\mathrm{I}(\mathbf{d}) / \mathrm{I}(\mathbf{c})=\left\{\Delta /\left[\mathrm{A}+\left(\mathrm{A}^{2}+\Delta^{2}\right)^{1 / 2}\right]\right\}^{2}$

The rate of relaxation by incoherent tunnelling from the $\mathrm{L}$ to the $\mathrm{R}$ tautomer 

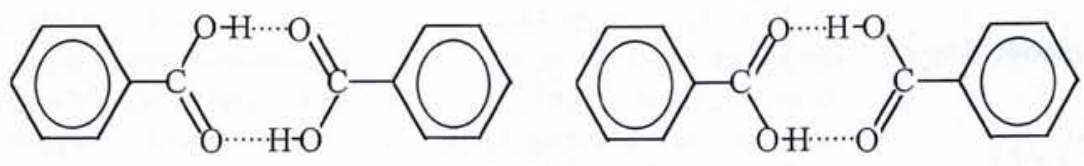

L

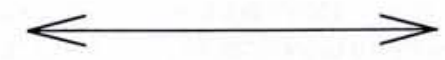

$\mathrm{R}$
Fig 2 The interchange between the two tautomers of benzoic acid dimers is mediated by a double protontransfer in the hydrogen bonds. At low temperature these dynamics are dominated by translational quantumtunnelling is determined by resolving the

fluorescence intensity corresponding to the $\mathrm{L}$ and $\mathrm{R}$ tautomers as a function of time subsequent to selective pulsed excitation of the dye at the frequency of the transitions $\mathrm{a}, \mathrm{b}$, which populate the $\mathrm{L}$ tautomer only. These measurements have been made with a number of different dye molecules and depend only slightly on the nature of the dye. The numbers

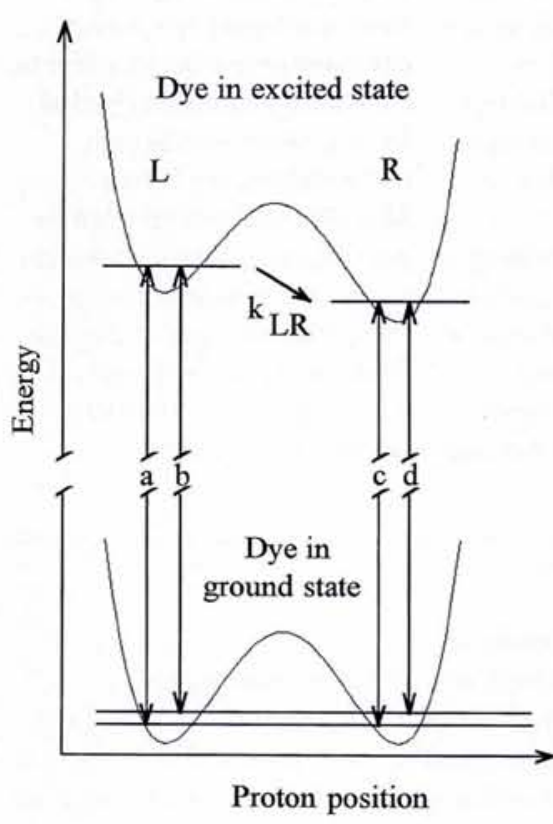

Fig 3 Three diagrams above Illustration of the optical spectroscopic methods used to resolve the tunnelling level structure and the relaxation dynamics of benzoic acid dimers. The absorption and fluorescence spectra of a dye make it possible to determine the tunnelling level splitting and the relaxation dynamics of a neighbouring benzoic acid dimer, weakly coupled to the dye molecule indicated in fig $3, \Delta=8 \mathrm{GHz}$ and

$\mathrm{k}_{\mathrm{LR}}=5 \times 10^{8} \mathrm{~s}^{-1}$, are typical values. The increase of the homogeneous linewidth of electronic transitions of dyes in condensed phases, restricts these optical spectroscopic measurements to low temperatures $(<15-20 \mathrm{~K})$.

At higher temperatures, the proton correlation time, $\tau_{c}$, defined by $1 / \tau_{c}=k_{L R}+k_{R L}$, was determined from
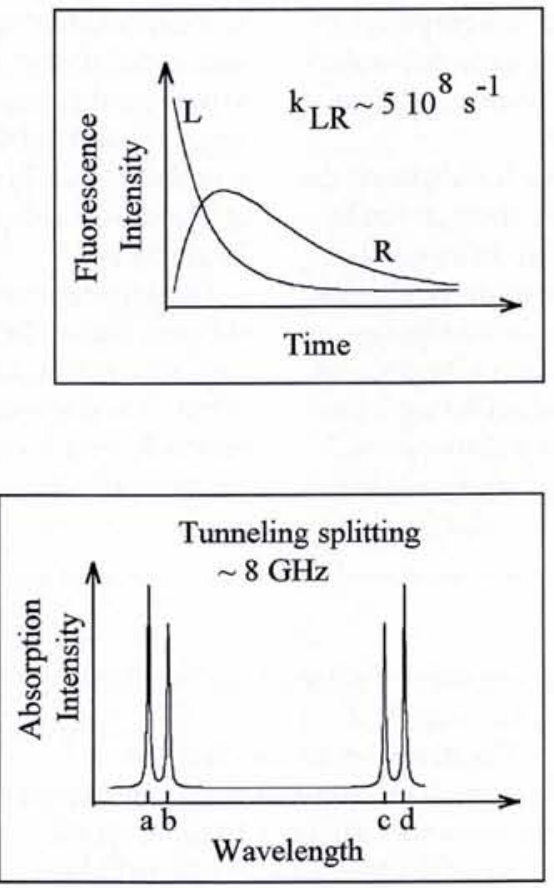

NMR and QENS [4-9]. Of particular interest is the temperature region in which other thermally activated processes begin to contribute to the proton transfer and mark the onset of the transition from pure quantum mechanical to classical barrier crossing. This region was characterized by measurements of the proton correlation time using QENS on the IN16 spectrometer at the Institut LaueLangevin. After scattering on the sample, the neutrons of an incident monochromatic beam acquire an energy uncertainty and a so-called quasi-elastic signal is recorded. The inverse of the quasi-elastic linewidth equals the proton correlation time $\tau_{c}$. Because of the restricted energy transfer window of $\mathrm{IN}_{16} 6$ and because the intensity of the quasielastic line is a function of temperature, depending on the population of the higher energy potential well, hydrostatic pressure of $2 \mathrm{kbar}$ was applied to the sample. Hydrostatic pressure decreases the energy asymmetry which has the effect of populating the higher energy well. Furthermore, the overlap of the localized states is enhanced and a larger tunnelling matrix element results. Since $1 / \tau_{c}$ scales with $\Delta^{2}$, the quasi-elastic linewidth, characterizing the transition region, could be brought within the energy transfer window of the IN16 spectrometer.
Fig 4 Right The proton transfer rate, $\tau_{c}^{-1}$, in benzoic acid as a function of temperature at a pressure of 2 kbar. (QENS: squares, NMR: circles). The data asymptotically approach the incoherent quantum-tunnelling regime at low temperature and the classical barrierhopping regime at high temperature. The lines are fits to theory, the solid line includes the contribution of tunnelling in both ground and first excited vibrational states

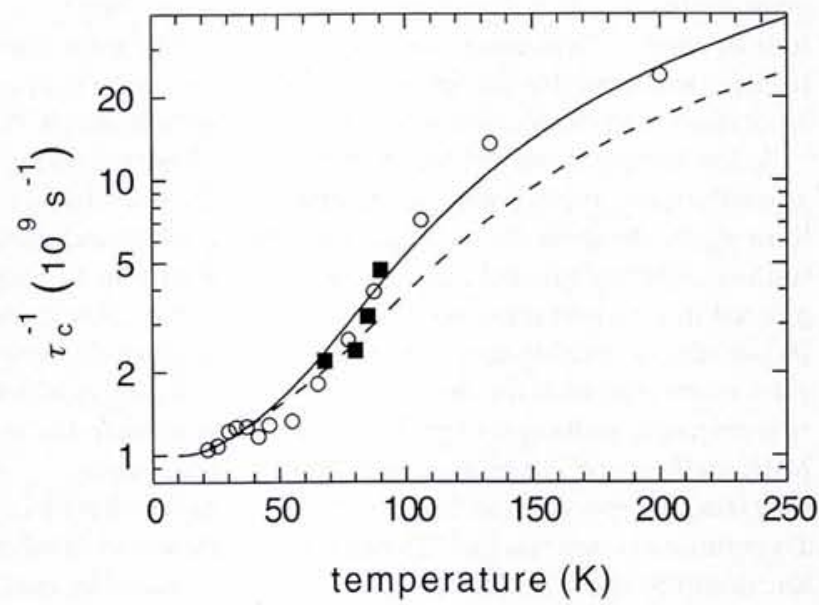




\section{And All That, Thomson's Finest Hour}

\section{Or was it?}

What with all the exhibitions, articles in magazines (naturally including this one), conferences and Websites on the subject this year it would be easy to get the impression that Joseph John Thomson discovered the electron. Cambridge University, which was Thomson's home from home one-hundred years ago, has little doubt of the fact. But Franklin is not so sure.

'I cannot quite agree', said Abraham Pais in his splendid history of modern physics, Inward Bound.

Indeed, Irish physicists point to George Stoney who named the electron in 1891. But he had in mind an elementary quantum of charge rather than a particle. The French give some precedence to Jean Baptiste Perrin in their version of popular scientific folklore

(See Sciences et Avenir, May 1997, p 40). Others might claim that the Zeeman effect (1896), as interpreted with the electron theory of Dutchman Hendrik Lorentz, gave a measurement of elm of comparable accuracy to that of Thomson. Then there were Wiechert and Kaufmann. Let's face it, the electron was dansle vent in more places than the Cavendish Laboratory by the time Thomson did his experiment.

The excellent Website on the history of the electron run by our American cousins at the American Institute of Physics credits Thomson with proposing the idea of the electron, rather than discovering it one afternoon in his laboratory. It points out it took a good few experiments before the idea was accepted. But like most histories of the electron it leaves out one interesting point, that Heinrich Hertz had already shown cathode rays were not deflected by an electric field and so were not particulate (great men never get everything right). It must have been irksome for the Germans to have a British physicist prove otherwise. Discoveries were never free from the geography of politics (and aren't today).

Those fortunate enough to teach undergraduates have to be ruthless in simplifying history, if they do not ignore it altogether. Such surgery, when applied to the tangled tales of research, is acceptable so long as it does not have a persistent bias, as Sciences et Avenir seems to have (in this case). Then again, the story of the electron is probably usually an example of 'l'histoire écrite par des Anglo-Saxons'.

Franklin has been listening to Denis Weaire, EPS president - a physicist with a fondness for history - and agrees wholeheartedly with his comments on the matter: 'The questioning of myth and the reassessment of precedent and prejudice can help us to show the way forward as well as to illuminate the past.'

Whenever doubt arises we need an informed debate on the historiography of our subject which recognizes the fascinating complexity of the process of discovery, which is usually communal rather than individual. The remarkable Thomson could hardly be diminished by putting him in context in this way. Pais draws attention to his identification of photoelectrically produced particles as electrons, in 1899 , calling this his 'finest hour as an experimentalist'.

\section{Do you agree with Franklin? Do you have anything to add? Send your replies by e-mail to eneditor@univ-mulhouse.fr or to Franklin, c/o European Physical Society, 34 rue Marc Seguin, F-68060 Mulhouse, France.} All replies will be considered for publication. Visit the AIP's electron history Website at www.aip.org/ history/electron where you can hear Thomson's voice. Then try IOP's at www.iop.org/Physics/Electron/ Exhibition for a movie clip. from page $141 \quad$ In fig 4 the filled squares are the QENS measurements of $1 / \tau_{c}$ as a function of temperature. These measurements enable us to calibrate NMR measurements of $\mathrm{T}_{1}$ at the same pressure. The proton transfer rates extracted from this analysis are shown as open circles. Figure 4 illustrates the quantum-to-classical transition for proton transfer in the double minimum potential associated with the hydrogen bond.

At low temperature, the behaviour asymptotically approaches the quantum limit where the dynamics are governed by tunnelling in the ground state. Extrapolated to zero pressure, the rate of incoherent tunnelling agrees with the values obtained with the optical spectroscopic techniques and by novel NMR methods [9]. At high temperature, the classical limit (barrier hopping) is asymptotically approached. The dashed line is a fit to theory where simple interpolation between the two limiting cases is applied.

The deviation of this fit in the intermediate temperature region exposes the enhancement of tunnelling via the thermal excitation of vibrational levels involving modes which promote tunnelling.

The solid line in fig 4 represents a fit including only one excited vibrational state in which $\Delta$ is increased to $\Delta^{\prime}=4.5 \Delta$. These measurements not only represent the first characterization of proton transfer in hydrogen bonds in the quantum-to-classical transition region, but are also important in validating the analysis of conventional and novel NMR techniques which can be more generally applied to the study of these fundamental processes.

This article first appeared in ILL's Annual Report 96. It was updated by the authors and reprinted by kind permission of the editors.

\section{References}

[1] G.R. Holtom, R.M. Hochstrasser, H.P. Trommsdorff, Chem. Phys. Lett. 131 (1986) 44 [2] Ch. Rambaud, A. Oppenländer, M. Pierre, H.P. Trommsdorff, J.C. Vial, Chem. Phys. 136 (1989) 335 [3] A. Oppenländer, Ch. Rambaud, H.P. Trommsdorff, J.C. Vial, Phys. Rev. Letters, 63 (1989) 1432.

[4] H.P. Trommsdorff, M. Johnson, M. Neumann, L. von Laue, D.F. Brougham, A.J. Horsewill, Nato ASI Series, Kluwer Academic Publishers, Dordrecht, 1997, pp. 369.

[5] A. Stöckli, B.H. Meier, R. Kreis, R. Meyer and R.R. Ernst, J.Chem.Phys. 93 (1990) 1502

[6] A.J. Horsewill and A. Ikram, Physica B. 226 (1996) 202

[7] A. Stöckli, A. Furrer, Ch. Schönenberger, B.H. Meier, R.R. Ernst and I. Anderson, Physica B 136 (1986) 161

[8] A.J. Horsewill, P.J. McDonald and D. Vijayaraghavan, J.Chem.Phys. 100 (1994) 1889. [9] D.F. Brougham, A.J. Horsewill and R.I. Jenkinson, Chem.Phys.Letters, in press 